\title{
El comportamiento del ciudadano organizativo
}

\section{The behavior of the organizing citizen}

\author{
María Auxiliadora Guerrero Bejarano, MBA \\ Universidad Internacional del Ecuador, Ecuador \\ Daniel Ricardo Silva Siu, Msc \\ Universidad ESAN, Perú
}

Autor por Correspondencia: maguerrerobe@uide.edu.ec, dsilva@pobox.com

Fecha de recepción: 6 de Marzo de 2017 - Fecha de aceptación: 5 de Junio de 2017

\begin{abstract}
Resumen
Las organizaciones son instituciones conformadas por personas que suelen tener un objetivo común, pero aunque en general todas cumplen con este principio, no siempre consiguen los mismos resultados, desde hace algún tiempo los académicos vienen buscando analizar el porqué de esta diferencia, y debido a esto se ha estudia desde hace décadas los diferentes factores que podrían influir en el desempeño de las organizaciones. Entre estos factores se encuentra el comportamiento del ciudadano organizativo, nombre con el que se conoce al miembro de la organización qué generalmente demuestra mayores niveles de compromiso con la misma, este análisis teórico busca describir de manera teórica la definición de este miembro quién puede influir positivamente en los resultados obtenidos por la empresa.
\end{abstract}

Palabras Clave: organizaciones; factores; compromisos

\begin{abstract}
The organizations are institutions made up of people who usually have a common goal, but although in general all comply with this principle, do not always achieve the same results, for some time academics have been trying to analyze the reason for this difference, and due to This has been studied for decades the different factors that could influence the performance of organizations. Among these factors is the behavior of the organizational citizen, the name with which the member of the organization is known which generally demonstrates higher levels of commitment to it, this theoretical analysis seeks to describe in a theoretical way the definition of this member who can influence positively In the results obtained by the company.
\end{abstract}

Key words: organizations; factors; commitments 


\section{Introducción}

\section{Comportamiento del Ciudadano Organizativo}

El comportamiento del ciudadano organizativo, u organizacional se ha definido desde la década de los 60, donde diferentes autores lo han descrito como la disposición de los individuos para contribuir con la organización, uniendo esfuerzos y haciendo que prevalezca la actitud de equipo (Harper, 2015; Organ, 1990), así también identifican factores que caracterizan este comportamiento como (a) la necesidad de inducir y mantener a los miembros de la organización en el sistema, (b) tener roles definidos y propios con dependencia y (c) permirtir a las personas ser espontáneas e inyectar innovación al rol que ejercen (Katrinli, Atabay, \& Gunay, 2006). Se define entonces como la conducta discrecional y adicional que no hace parte de los requisitos esperados o formales de un puesto, pero que promueven el funcionamiento eficaz de la organización (D'INTINO, SHEPARD, \& WOLFLE, 2002). El Comportamiento del Ciudadano Organizacional (CCO) implica diversos comportamientos "espontáneos/no obligatorios" que no suelen aparecer en las descripciones de los puestos de trabajo, pero que son valorados por los líderes dados su impacto positivo en la organización. El CCO no es un comportamiento único sino debe ser entendido como un concepto multidimensional, que incluso guarda cercanía teórica con otros constructos, generalmente es relacionado con la satisfacción laboral, el compromiso laboral y el estilo de liderazgo ejercido por los jefes (Chiaburu \& Baker, 2006; Dimitriades, 2007; Foote \& Li-Ping Tang, 2008; Moideenkutty, Blau, Kumar, \& Nalakath, 2005; Piercy, Lane, \& Cravens, 2002; Saks, 2006; Xenikou \& Simosi, 2006). EL CCO se correlaciona positivamente con el liderazgo transformacional (Dexter \& Prince, 2007) y un clima organizacional que alienta la innovación . El CCO es una variable dependiente al igual que la productividad, satisfacción laboral, rotación y ausentismo (Becton, Giles, \& Schraeder, 2008; MacKenzie, Podsakoff, \& Fetter, 1991; Zoghbi Manrique de Lara \& Espino Rodríguez, 2007).

Se define al CCO como un constructo multidimensional a la hora de ser medido, sus dimensiones son: cortesía, tolerancia, respeto, iniciativa, lealtad, altruismo y ayuda. Para algunos autores debe ser medido únicamente con dos factores, altruismo (capacidad de ayuda directa e intencional cara a cara) y la conformidad generalizada, que se define como la capacidad de los individuos para aceptar e internalizar las reglas y procedimiento de la empresa (Dyne, Ang, \& Botero, 2003). Para Organ (1990) el constructor posee 5 factores: altruismo, cortesía, consciencia, virtud cívica y la capacidad para tolerar inconvenientes, este autor incluye un sexto factor: hacer la paz. Otros autores determinan que la multidimensión del constructo se encuentra definida por dos factores, el CCO individual que contiene el altruismo y la cortesía de los miembros de la organización hacia otros miembros y el CCO organizacional que contendría la conciencia, la virtud cívica y la capacidad para tolerar inconvenientes que estarían dados por la cultura organizacional (Bolon, 1997; Ma, Qu, Wilson, \& Eastman, 2013; Somech \& DrachZahavy, 2004; Werner, 2000). Este constructo también se define para otros con siete factores: ayudar, tolerar inconvenientes, la lealtad organizacional, la conformidad organizacional, la iniciativa individual, la virtud cívica y el autodesarrollo (Coyle-Shapiro \& Kessler, 2000; Kamdar, McAllister, \& Turban, 2006; Ong, 2012). Existen muchas coincidencias en las diferentes definiciones del constructo, que lo que esperan es describir las diferentes características del comportamiento de los miembros de una organización y su predisposición a ayudar a los demás para obtener los propósitos generales de la misma. 
Existen estudios que han analizado si existiría una influencia de género en el desarrollo del comportamiento del ciudadano organizacional, así también la influencia del género en dicho comportamiento de acuerdo con la tarea o rol que desempeña el empleado (Piercy et al., 2002).

A partir de estas ideas podría parecer lógico aceptar que otros constructos, como la satisfacción laboraría son determinantes para el comportamiento del ciudadano organizativo; se esperaría que los empleados satisfechos sean más proclives a hablar en forma positiva acerca de la organización, así como a ayudar a otros a conseguir mejores resultados o a ir más allá de las expectativas de su puesto, tal vez por un deseo a ser recíprocos por las experiencias positivas que han tenido dentro de la organización (Foote \& Li-Ping Tang, 2008; Robbins, Judge, Millett, \& Boyle, 2013). Así mismo el nivel de compromiso de los empleados tendría una relación con el comportamiento de estos dentro de la organización y su predisposición para dar algo más de lo esperado en el desempeño en su puesto de trabajo (Saks, 2006).

El desarrollo del CCO previene costosas intervenciones o situaciones de bajo rendimiento; la correcta selección de los ciudadanos adecuados puede reducir los costos de capacitación, desempeño y rotación. Una buena orientación y capacitación mejoran la calidad y la satisfacción del cliente, los equipos de trabajo cohesivos que emplean sólidos procesos de medición y gestión del rendimiento infunden altos valores en el trabajo diario. La planificación sabia y la implementación nítida vinculan la misión, la estrategia y el rendimiento en un todo sin fisuras. El modelo de ciudadanía ofrece una variedad de oportunidades para la investigación organizacional futura; por lo que podríamos afirmar que el comportamiento del ciudadano organizacional también afectaría la forma de aprendizaje de la organización, así como el manejo del conocimiento (Brightman \& Moran, 1999). Un estudio previo analizó no solo el estilo de liderazgo como factor que influye en el desarrollo del CCO, sino también el nivel de confianza presente en la organización, y encontró que aunque no había un nivel alto de significancia, sí existía una influencia del estilo de liderazgo y el nivel de confianza presente en el tipo de comportamiento de los ciudadanos organizativos, así también que la insatisfacción laboral podría afectar el comportamiento (Appelbaum et al., 2004). Las investigaciones indican que la OCB influye no sólo en las evaluaciones de rendimiento subjetivas, sino también en las decisiones de asignación de recompensas (Podsakoff, MacKenzie, Paine y Bachrach, 2000).

Investigaciones previas muestran que incluso en entornos sindicalizados, los supervisores toman en consideración el CCO para evaluar el desempeño. Se asume que el CCO afecta las decisiones de asignación de recompensas en este tipo de entornos, debido a que generalmente los sindicatos asignan recompensas en base a la antigüedad o medidas objetivas de desempeño (Moideenkutty et al., 2005).

\section{Conclusión}

Se entiende que el éxito de cada empresa se debe al desempeño y buen trabajo de sus empleados, algunos autores recomiendan mirar a las organizaciones como países, dónde se crea la ciudadanía organizacional como un compromiso voluntario y coherente con los objetivos, métodos y el éxito de la empresa; entendiendo que las estrategias que los jefes utilizan para liderar a sus equipos influirán en la creación de esta ciudadanía, y en el nivel de patriotismo presente en la organización (Brightman \& Moran, 1999). 
Hay estudios que demuestran la existencia de una diferencia significativa entre los compromisos del trabajo y la organización y que el apoyo percibido de la organización predice tanto el trabajo como la participación de la organización; las características del trabajo predicen la participación en el trabajo; y la justicia procesal predice el compromiso de la organización (Saks, 2006). Es importante entonces que se considere los diferentes factores que permiten el desarrollo del comportamiento en la ciudadanía organizacional, puesto que puede ser un correctivo ante la preocupación que puede existir en la dirección con respecto al estilo de liderazgo aplicado y los resultados obtenidos en los miembros del equipo. Al definir la buena gestión como la creación de procesos operativos que refuercen el compromiso y la contribución al éxito, los líderes pueden aprovechar los mejores talentos de su gente al servicio de las prioridades de la organización. (Brightman \& Moran, 1999).

La identificación del CCO es una herramienta que permite a los gerentes reconocer entre sus miembros habilidades que pudieran llevarlos a desempeñar roles de liderazgo, así como a entender cómo los diferentes aspectos de la personalidad de cada miembro deben hacer parte de la forma en la que se desafía su desarrollo dentro de la empresa (Chiaburu \& Baker, 2006). Los empleados deben tener conciencia de la importancia de su comportamiento, debido a que este hará parte de la evaluación que los jefes o gerentes hacen en determinados momentos, de no ser así, puede existir una percepción equivocada por parte de los empleados que podría desmotivar al trabajador y hacerlo perder la confianza hacia el sistema de evaluación y su imparcialidad (Moideenkutty et al., 2005). Si los gerentes consideran que el CCO es importante, deben tomar medidas para promover estos comportamientos. Las investigaciones indican que la OCB se mejora cuando los empleados son tratados de manera justa, cuando tienen buenas relaciones con sus supervisores y cuando sus puestos de trabajo les proporcionan autonomía y desafío. Estos hallazgos proporcionan amplio margen para que los gerentes diseñen prácticas específicas que mejoren la OCB entre sus empleados (Moideenkutty et al., 2005).

\section{Bibliografía}

Appelbaum, S., Bartolomucci, N., Beaumier, E., Boulanger, J., Corrigan, R., Dore, I., ... Serroni, C. (2004). Organizational citizenship behavior: A case study of culture, leadership and trust. Management Decision, 42(1), 13-40.

Becton, J. B., Giles, W. F., \& Schraeder, M. (2008). Evaluating and rewarding ocbs. Employee Relations, 30(5), 494.

Bolon, D. S. (1997). Organizational citizenship behavior among hospital employees: A multidimensional analysis involving job satisfaction and organizational commitment. Journal of Healthcare Management, 42(2), 221.

Brightman, B. K., \& Moran, J. W. (1999). Building organizational citizenship. Management Decision, 37(9), 678-685. 
Chiaburu, D. S., \& Baker, V. L. (2006). Extra-role behaviors challenging the status-quo: Validity and antecedents of taking charge behaviors. Journal of Managerial Psychology, 21(7), 620-637.

Coyle-Shapiro, J., \& Kessler, I. (2000). Consequences of the psychological contract for the employment relationship: A large scale survey. Journal of management studies, 37(7), $903-$ 930.

D'INTINO, R. S., SHEPARD, J. M., \& WOLFLE, L. M. (2002). A social contract perspective on organizational citizenship behavior. Paper presented at the Academy of Management Proceedings.

Dexter, B., \& Prince, C. (2007). Evaluating the impact of leadership development: A case study. Journal of European industrial training, 31(8), 609-625.

Dimitriades, Z. S. (2007). The influence of service climate and job involvement on customeroriented organizational citizenship behavior in greek service organizations: A survey. Employee Relations, 29(5), 469-491.

Dyne, L. V., Ang, S., \& Botero, I. C. (2003). Conceptualizing employee silence and employee voice as multidimensional constructs. Journal of management studies, 40(6), 1359-1392.

Foote, D. A., \& Li-Ping Tang, T. (2008). Job satisfaction and organizational citizenship behavior (ocb) does team commitment make a difference in self-directed teams? Management Decision, 46(6), 933-947.

Harper, P. J. (2015). Exploring forms of organizational citizenship behaviors (ocb): Antecedents and outcomes. Journal of Management and Marketing Research, 18, 1.

Kamdar, D., McAllister, D. J., \& Turban, D. B. (2006). " All in a day's work": How follower individual differences and justice perceptions predict ocb role definitions and behavior. Journal of applied psychology, 91(4), 841.

Katrinli, A. E., Atabay, G., \& Gunay, G. (2006). Congruence of family and organizational values in relation to organizational citizenship behaviour. Journal of human values, 12(1), 81-89.

Ma, E., Qu, H., Wilson, M., \& Eastman, K. (2013). Modeling ocb for hotels: Don't forget the customers. Cornell Hospitality Quarterly, 54(3), 308-317.

MacKenzie, S. B., Podsakoff, P. M., \& Fetter, R. (1991). Organizational citizenship behavior and objective productivity as determinants of managerial evaluations of salespersons' performance. Organizational behavior and human decision processes, 50(1), 123-150.

Moideenkutty, U., Blau, G., Kumar, R., \& Nalakath, A. (2005). Relationship of organization citizenship behavior and objective productivity to managerial evaluations of performance in india. International Journal of Commerce and Management, 15(3/4), 221-229. 
Ong, L. D. (2012). Employees' job performance at the workplace. Actual Problems of Economics, $137(11), 418-424$.

Organ, D. W. (1990). The motivational basis of organizational citizenship behavior. Research in organizational behavior, 12(1), 43-72.

Piercy, N. F., Lane, N., \& Cravens, D. W. (2002). A gender perspective on salesperson organizational citizenship behaviour, sales manager control strategy and sales unit effectiveness. Women in Management Review, 17(8), 373-391.

Robbins, S., Judge, T. A., Millett, B., \& Boyle, M. (2013). Organizational behaviour: Pearson Higher Education AU.

Saks, A. M. (2006). Antecedents and consequences of employee engagement. Journal of Managerial Psychology, 21(7), 600-619.

Somech, A., \& Drach-Zahavy, A. (2004). Exploring organizational citizenship behaviour from an organizational perspective: The relationship between organizational learning and organizational citizenship behaviour. Journal of occupational and organizational psychology, 77(3), 281-298.

Werner, J. M. (2000). Implications of ocb and contextual performance for human resource management. Human resource management review, 10(1), 3-24.

Xenikou, A., \& Simosi, M. (2006). Organizational culture and transformational leadership as predictors of business unit performance. Journal of Managerial Psychology, 21(6), 566579 .

Zoghbi Manrique de Lara, P., \& Espino Rodríguez, T. F. (2007). Organizational anomie as moderator of the relationship between an unfavorable attitudinal environment and citizenship behavior (ocb) an empirical study among university administration and services personnel. Personnel Review, 36(6), 843-866. 